\title{
Diffuse Interstellar Lines and Chemical Characterization of Interstellar Dust
}

\author{
Fred M. JoHnson \\ Electro-Optical Systems, Inc. \\ Pasadena, California
}

$\mathrm{T}$

WE PROBLEM OF CHEMICAL IDENTIFICATION of the interstellar dust grains has vexed astronomers for over three decades. The earliest evidence for the mere presence of interstellar dust was deduced from the observation of stars (via star counts) in the Milky Way. The best presently available clues for possible chemical identification are spectroscope data, particularly the diffuse (hitherto unidentified) interstellar absorption lines. This paper is concerned primarily with a discussion of new theoretical interpretations of the spectroscopic data.

Much related information on the dust grains was obtained from observations of space reddening, its wavelength dependence, and from interstellar polarization. Furthermore, correlation of space reddening and the strength of some of the diffuse lines is important to the discussion of possible chemical identification of the dust.

The observed interstellar polarization requires asymmetrically shaped dust grains, and, according to the theory of Davis and Greenstein, they must be paramagnetic. References 1 to 24 contain much pertinent information on various aspects of the grain problem. An excellent introduction to the field is given in reference 1 , and an extensive bibliography is found in reference 5 . The chemical problems are discussed in reference 13; experiments on trapped radicals are described in reference 6; and a recent article (ref. 10) revives the graphite particle hypothesis. Theoretical discussions on the formation of dust particles and on other related topics on the interstellar medium are given in references 19 to 22. The fact that all identified interstellar molecular lines involve a carbon atom ( $\mathrm{OH}$ excepted) makes it highly likely that grains could wholly or partly involve carbon.

Among the properties which these grains would have to satisfy are:

1. They are probably elongated dielectric particles (or semiconductors). 
2. Their average size is about $10^{-5} \mathrm{~cm}$.

3. Their density in space is between $10^{-25}$ and $10^{-26} \mathrm{~g} / \mathrm{cm}^{3}$.

4. The fact that they are paramagnetic follows from the fact that the polarization of starlight requires them to be oriented in the galactic magnetic field.

Of particular significance among the interstellar lines are the rather wide diffuse bands, mainly those at $4430 \AA, 4760 \AA, 4890 \AA$, and $6180 \AA$ (ref. 14), and others recently discovered by G. H. Herbig. Table I lists the known theories on the identification of the diffuse lines. An abbreviated description of these theories and some remarks are included.

TABLE I. -Suggestions for Identification of Interstellar Lines

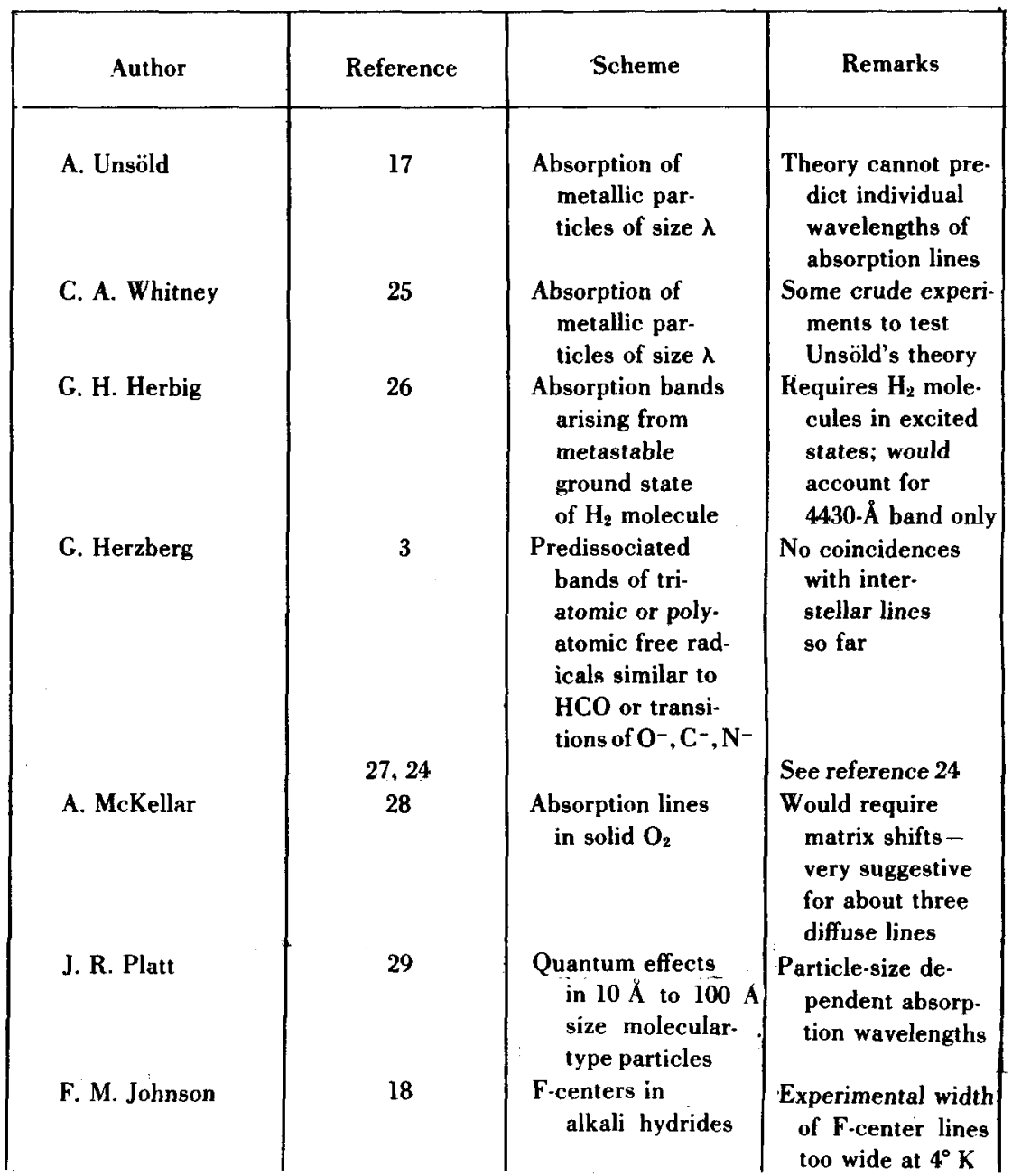


TABLE I. - Suggestions for Identification of Interstellar Lines - Concluded

\begin{tabular}{|c|c|c|c|}
\hline Author & Reference & Scheme & Remarks \\
\hline $\begin{array}{c}\text { E. E. Ferguson } \\
\text { and }\end{array}$ & 30 & $\begin{array}{c}\text { Molecular complex } \\
\text { charge transfer } \\
\text { H. P. Broida } \\
\begin{array}{c}\text { P. Swings and } \\
\text { M. Desirant }\end{array}\end{array}$ & $\begin{array}{c}\text { No coincidence } \\
\text { found so far }\end{array}$ \\
$\begin{array}{c}\text { P. Swings and } \\
\text { Y. Öhman }\end{array}$ & 31 & $\begin{array}{c}\text { General discussion } \\
\text { on absorption in } \\
\text { spectra in solids } \\
\text { at low } \\
\text { temperatures }\end{array}$ \\
F. M. Johnson & Present \\
compilation & $\begin{array}{c}\text { Suggest molecular } \\
\text { crystals and } \\
\text { amorphous metals }\end{array}$ & $\begin{array}{c}\text { Hydrocarbons } \\
\text { Frequency analysis } \\
\text { of all interstellar } \\
\text { lines could be } \\
\text { fitted to a simple } \\
\text { pattern }\end{array}$ \\
\hline
\end{tabular}

\section{MODELS FOR VIBRATIONAL ANALYSIS}

A frequency analysis was made of the 18 interstellar diffuse bands provided by Dr. G. H. Herbig. It was found that these bands could be arranged in groups as shown in figure 1.

It should be noted that some of these energy differences are multiples of a mean value, an indication of the possibility for as yet undiscovered interstellar features. However, the absence of such missing lines does not necessarily invalidate this theory since variations of intensities of molecular lines are well known. (See ref. 34.)

Distinctive but previously unrecognized patterns corresponding to vibrational energy separations within electronic bands have been identified among the 18 diffuse interstellar lines. Each of the 18 lines falls into one of four groups. The first group contains two subgroups which occur in the vicinities of $4600 \AA$ and $6100 \AA$, respectively, the former being the more diffuse. Both of these subgroups comprise a set of 3 lines with wavenumber separations of $556 \mathrm{~cm}^{-1}$ and $1568 \mathrm{~cm}^{-1}$. The remaining 12 lines fall within a third group, also near $6100 \AA$, whose mean wavenumber separations can be sorted into $226 \mathrm{~cm}^{-1}$ or multiples thereof.

Since a vibrational analysis was thought to be suggestive of vibrational structure associated with complex molecules, the following models were considered: electronic-vibration transitions and inverse Raman effect. This latter phenomenon shows some interesting coinci- 


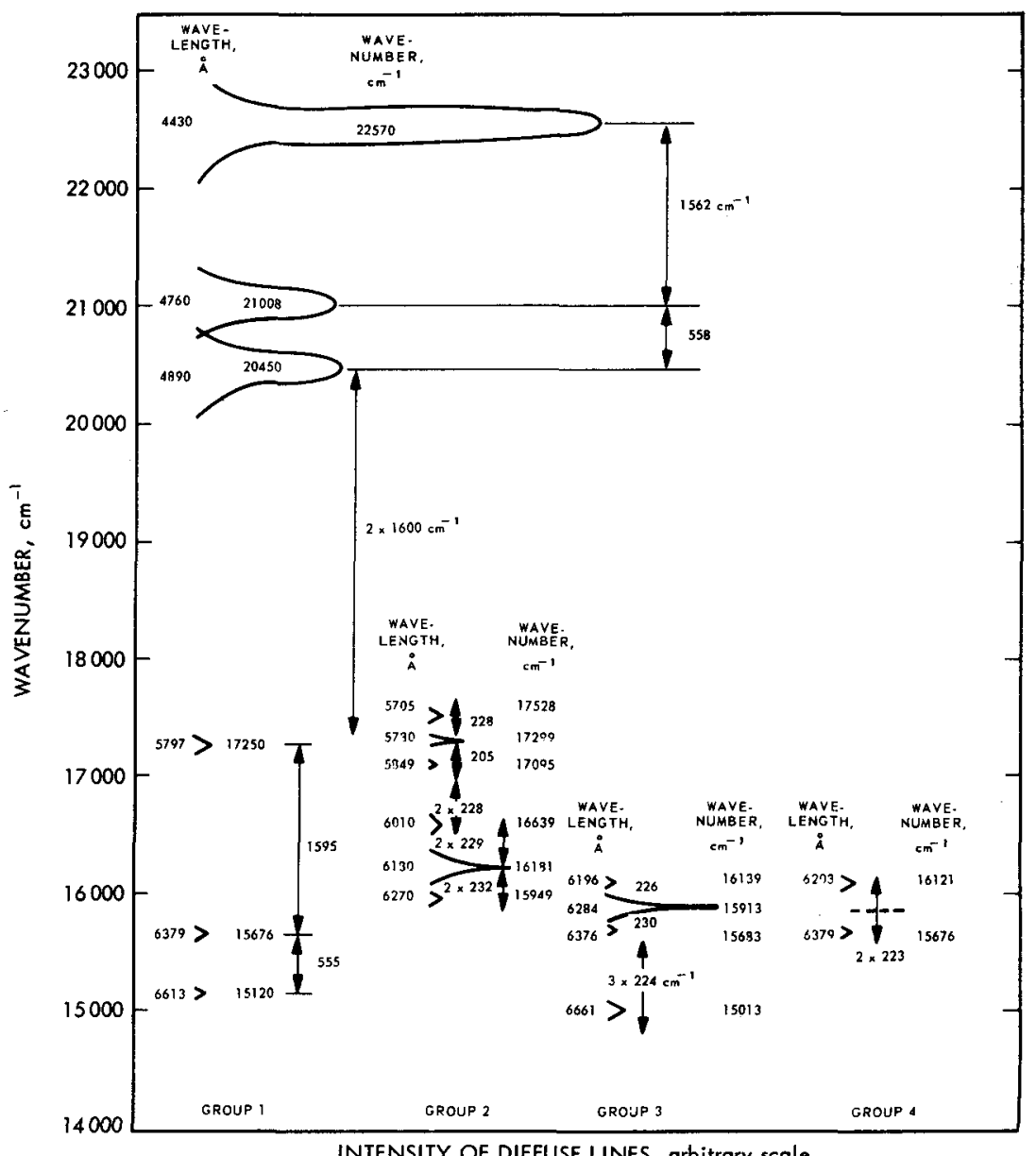

FIGURE 1. - Relative intensities and wavelengths of diffuse lines.

dences, and was, in fact, independently considered by B. P. Stoicheff; however, it is thought to be unlikely for several reasons discussed later.

\section{Electronic-vibration transitions}

If electronic-vibrational transitions are responsible for producing diffuse interstellar features, one requires transitions from the ground state to various excited states in these complex molecules or radicals. Examination of the literature of molecular spectroscopy reveals many hydrocarbons of the type $\mathrm{C}_{x}-\mathrm{H}_{y}-\mathrm{N}_{z}$ having a large variety of vibrational frequencies close to those of interest. Some examples are $\mathrm{C}_{2} \mathrm{~N}_{2}$ and diacetylene having vibrational frequencies of 226 and 231 wavenumbers, respectively, for the lowest electronic-vibrational states. Note that vibrational frequency in the excited states of the molecular 
radicals is desired. It is possible that a variety of similar compounds embedded or associated with these grains may be involved. Thus one can explain the difference in the widths of the broad lines compared with the narrow lines of figure 1 . A large selection of similar molecules would give a broader line, or the same set of molecules in different matrices might yield the diffuse lines $(4430 \AA, 4760 \AA$, and $4890 \AA)$. Since the strongest absorption lines are the widest, it seems more likely that a larger selection of molecules is the more likely explanation for the width and stronger intensity. The symmetric line shape of the absorption lines might rule against this hypothesis, however. The fact that no single molecule fits the observed vibrational lines exactly indicates that perhaps a selection of similar molecular vibrations are superimposed to give these broad features.

One interstellar line $(6379 \mathrm{~A})$ is used in the figure twice. It could either belong to the 225 or 555 wavenumber system. The fact that energy differences corresponding to a mean value of $225 \mathrm{~cm}^{-1}$ can be established among the remaining 9 interstellar lines indicates that a molecular vibrational frequency corresponding to this value plays a dominant role in the production of these features. In order to produce these molecular vibrational electronic excited states, either free radicals or a much larger type of molecular complex would have to be postulated. This analysis clearly shows that experimental work is necessary in order to make a definitive identification of these features. One of the severe experimental difficulties in attempting to duplicate these astronomical stellar features in the laboratory lies in the fact that low-temperature absorption studies in the solid state require substrates. These substrates can introduce "matrix" shifts of as much as $100 \AA$. (See ref. 35.)

\section{Inverse Raman effect}

The theory of this phenomenon is described in references 36 to 39 and has been demonstrated experimentally, as discussed in reference 40. The probability of absorption of a frequency $\nu_{0}$ and emission of $\nu_{1}$ where $\nu_{1}=\nu_{0} \pm \nu_{m}$ for $\nu_{m}$ an arbitrary frequency is given by

$$
\frac{16 \pi^{4}}{h^{4}} \int \mu^{2} \rho_{0}\left(\rho_{1}+\frac{8 \pi h \nu_{1}^{3}}{c^{3}}\right) \mathrm{d} \nu_{0}
$$

where $h$ is Planck's constant, $c$ is the velocity of light, and $\mu$ is the matrix element for the two-photon process. The energy density $\rho_{1}$, at $\nu_{0} \mp \nu_{m}$ is incident simultaneously with light at frequency $\nu_{0}$ and density $\rho_{0}$.

Although order-of-magnitude calculations indicate that the probability of a two-quantum process is extremely small, a discussion and a corresponding frequency analysis based on this model is included. This model requires the simultaneous irradiation of the interstellar grains by

271-992 O-67-16 

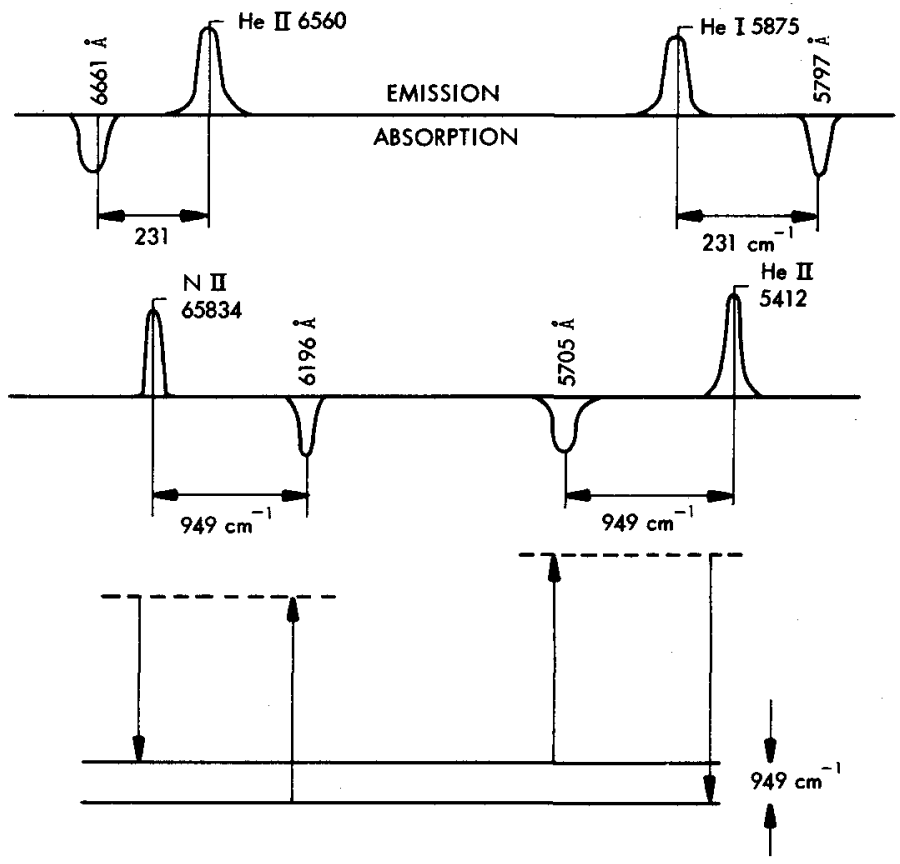

FIGURE 2. - Interstellar absorption and emission lines.
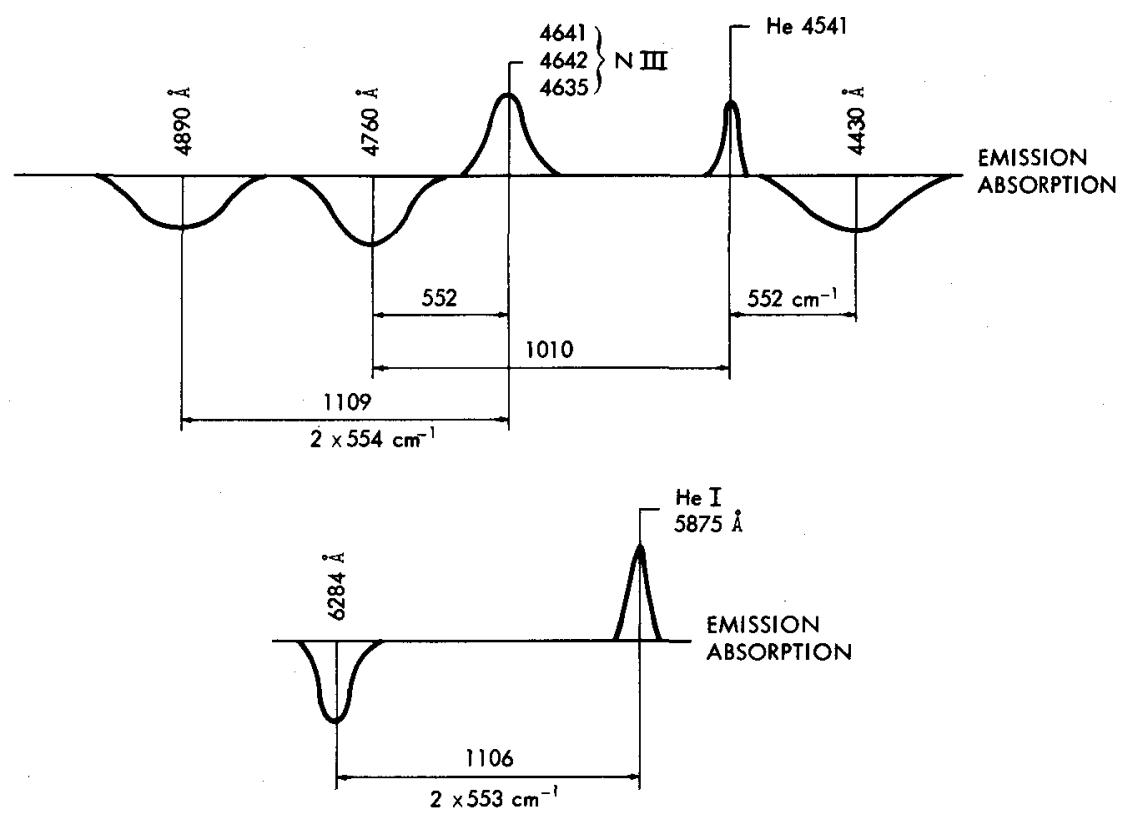

Figure 3.-Possible diffuse interstellar absorption lines. 


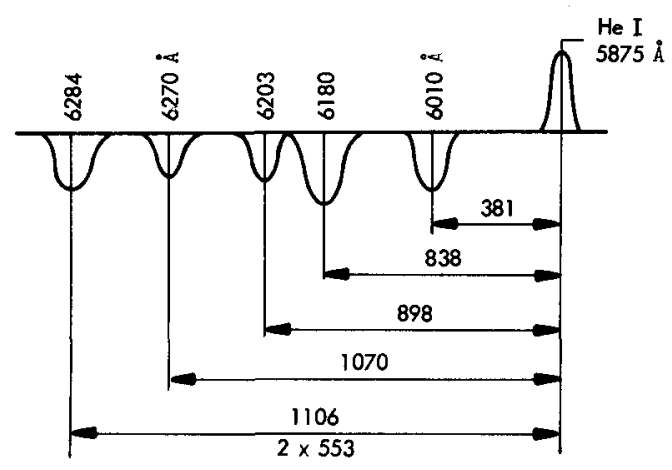

\begin{tabular}{|c|c|c|}
\hline ALLENE & $\mathrm{CH}_{2}=\mathrm{C}=\mathrm{CH}_{2}$ & $838,1071 \mathrm{~cm}^{-1}$ \\
\hline $\begin{array}{c}\text { CYCLOPENTENE } \\
\mathrm{C}_{5} \mathrm{H}_{8}\end{array}$ & & $386,896,1104 \mathrm{~cm}^{-1}$ \\
\hline $\begin{array}{c}\text { CYCLOPROPANE } \\
\mathrm{C}_{3} \mathrm{H}_{6}\end{array}$ & & $382,1022,1070,1120 \mathrm{~cm}^{-1}$ \\
\hline DIACETYLENE & $\mathrm{HC} \equiv \mathrm{C}-\mathrm{C} \equiv \mathrm{CH}$ & $231 v_{7}, \pi_{g^{\prime}}\left(v_{9}\right)$ \\
\hline $\mathrm{CH}_{3} \mathrm{CN}$ & $C-C \equiv N$ & $380 \mathrm{~s} v_{8}(e)$ BENDING VIBRATION \\
\hline
\end{tabular}

FIGURE 4.-Scheme for correlating "Stokes" absorption lines with an exciting source at $5875 \AA$.

emission lines $\left(\nu_{0} \pm \nu_{m}\right)$ as well as the usual stellar continuum. Both stellar as well as nebular emission lines were considered.

Because of the grain temperature $\left(\sim 4^{\circ} \mathrm{K}\right)$, all absorption transitions must arise from the ground state. Consequently, for both Stokes and anti-Stokes transitions to be observed, a pair of exciting sources are necessary.

Figures 2 and 3 show possible schemes satisfying these conditions. Figure 2 shows schematically the corresponding Raman transition through a virtual state. Figure 4 shows a possible scheme for correlating "Stokes" absorption lines with an exciting source at $5875 \AA$. With the exception of the $6284 . \AA$ line, all other absorption lines in figure 4 are inconsistent with the model unless corresponding anti-Stokes lines can be found. If this model were correct, it would be possible to identify these absorption features with well-known chemicals, such as allene, diacetylene, and a host of similar compounds. Table II lists some of these compounds with their pertinent vibrational frequencies. The width of 
the diffuse lines could be accounted for on this model by two possible schemes: (a) a variety of similar chemical species; and (b) width of exciting source, in particular the blend of three exciting lines $4641 \AA$, $4642 \AA$, and $4635 \AA$ comprising the N III source, which could account for the width of the wavelength $4430 . \AA, 4760-\AA$ and $4890-\AA$ diffuse lines.

Although more study is necessary to lend credence to the inverse Raman model, it is included here for completeness, particularly in view of the "pair coincidences" of a number of diffuse interstellar lines of figures 2,3 , and 4 .

TABLE II. - Vibrational Frequencies

\begin{tabular}{|c|c|}
\hline Compound & Vibrational frequencies, $\mathrm{cm}^{-1}$ \\
\hline $\mathrm{C}_{2} \mathrm{H}_{3} \mathrm{~N} \ldots \ldots \ldots \ldots \ldots \ldots \ldots \ldots \ldots \ldots \ldots \ldots \ldots \ldots \ldots \ldots$ & 380 \\
\hline $\mathrm{C}_{3} \mathrm{H}_{*}$ propane $\ldots \ldots \ldots \ldots \ldots \ldots \ldots$ & 375 \\
\hline $\mathrm{C}_{4} \mathrm{H}_{8}$ dimethylethene......... & 378 \\
\hline $\mathrm{C}_{2} \mathrm{H}_{8} \mathrm{~N}_{2}$ dimethylhydrazine....... & 949 \\
\hline $\mathrm{C}_{3} \mathrm{H}_{6} \mathrm{~N}$ dimethylcyanamid........ & $526,899,1105$ \\
\hline $\mathrm{C}_{3} \mathrm{H}_{5} \mathrm{~N}_{2} \ldots \ldots \ldots \ldots \ldots \ldots \ldots \ldots \ldots \ldots \ldots \ldots \ldots$ & 1010,829 \\
\hline $\mathrm{C}_{3} \mathrm{H}_{5} \mathrm{~N} \ldots \ldots \ldots \ldots \ldots \ldots \ldots \ldots \ldots \ldots \ldots \ldots \ldots \ldots \ldots$ & $384,545,220,1003,835,2245$ \\
\hline 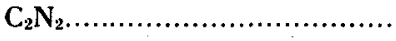 & $506,250,2336,2150$ \\
\hline
\end{tabular}

\section{CONCLUSION}

Preliminary results of a vibrational analysis of the diffuse interstellar lines is strongly suggestive of electronic-vibrational transitions in complex hydrocarbon molecules and/or radicals associated with the interstellar grain. Since none of the laboratory spectra so far fit the astronomical data, one is probably dealing with either very complex hydrocarbon molecules or simpler molecules in electronic states not ordinarily achieved under normal laboratory conditions, such as those embedded in specific matrices. Since it has been difficult to fit the optical astronomical absorption data, it is hoped that, as an intermediate step, the vibrational data might provide clues to the chemical identification.

\section{REFERENCES}

1. Greenstein, J. L.: Interstellar Matter. Astrophysics (J. A. Hynek, ed.), McGrawHill Book Co., Inc., 1951.

2. Dufay, J.: Galactic Nebulae and Interstellar Matter. Hutchinson, London, 1957.

3. Herzberg, G.: Laboratory Investigations of the Spectra of Interstellar and Cometary Molecules. Mem. Liège, vol. 15, 1955, p. 291.

4. DonN, B.: Survey of Theoretical and Experimental Research on Interstellar Grains. Lowell Obs. Bull., vol. 4, 1960, p. 273. 
5. DonN, B.: Trapped Radicals in Astrophysics. (A. M. Bass and H. P. Broida, eds.), Academic Press, 1960, pp. 347-362.

6. ANON.: Formation and Trapping of Free Radicals. (A. M. Bass and H. P. Broida, eds.), Academic Press, 1960.

7. Herbig, G. J.: Some Spectroscopic Problems of the Interstellar Medium. J. Quant. Spectr. Rad. Transfer, vol. 3, 1963, p. 529.

8. Bass, A. M.; and Brolda, H. P.: Absorption Spectra of Solids Condensed at Low Temperatures from Electric Discharges. Mol. Spectros., vol. 2, 1958, p. 42.

9. Platt, J. R.; and Donn, B.: Astron. J., vol. 61, 1956, p.11.

10. Hoyle, F.; and Wickramasinghe, N. C.: On Graphite Particles as Interstellar Grains. Roy. Astron. Soc., Monthly Notices, vol. 124, 1962, p. 417.

11. WiCkRAMasinghe, N. C.; and Guillaume, C.: Interstellar Extinction by Graphite Grains. Nature, vol. 207, 1965, p. 366.

12. Van de Hulst, H. C.: Light Scattering by Small Particles. John Wiley \& Sons, Inc., 1957.

13. DonN, B.: Some Chemical Problems of Interstellar Grains. Mem. Liège, vol. 15, 1954, p. 571.

14. Merrill, P. W.: Space Chemistry. Ann Arbor University Press, 1963.

15. Pikelner, S.: Soviet Science of Interstellar Space. Philosophical Library, New York, 1963:

16. Davis, L.; and Greenstein, J. L.: Polarization of Starlight by Aligned Dust Grains. Astrophys. J., vol. 114, 1951, p. 206.

17. Unsöld, A.: On the Interpretation of the Interstellar Absorption Bands. Z. Astrophys., vol. 56, 1963, p. 221.

18. Johnson, F. M.: Color Centers in Alkali Hydrides. Columbia University, Radiation Laboratory Progress Report, 1955, p. 30.

19. Kahn, F. D.: On the Formation of Interstellar Dust. Roy. Astron. Soc., Monthly Notices, vol. 112, 1952, p. 518.

20. SpITzer, L., JR.: Behavior of Matter in Space. Astrophys. J., vol. 120, 1954, p. 1.

21. BATES, D. R.; and SPITzer, L., JR.: The Density of Molecules in Interstellar Space. Astrophys. J., vol. 113, 1951, p. 441.

22. SPITZER, L., JR.; and Tukey, J. W.: A Theorv of Interstellar Polarization. Astrophys. J., vol. 114, 1951, p. 187.

23. Greenberg, J. M.: Interstellar Grains. Am. Rev. of Astron. and Astrophys., vol. 1, 1963, p. 267.

24. Henzererg, G.: Molecular Spectroscopy and Astrophysical Problems. J. Opt. Soc. Am., vol. 55, 1965, p. 229.

25. Whitney, C. A.: Exptl. Smithsonian Inst. Rept. No. 163, 1964.

26. Herisig, G. H.: The Diffuse Interstellar Bands. I. A Possible Identification of $\mathbf{1 4 4 3 0 .}$ Astrophys. J., vol. 137, 1963, p. 200.

27. Herzberg, G.: Publ. Roy. Obs. Edinburgh, vol. 4, 1964, p. 67.

28. McKellaR, A.: Astron. J., vol. 60, 1955, p. 170.

29. Platt, J. R.: On the Optical Properties of Interstellar Dust. Astrophys. J., vol. 123,1956 , p. 486.

30. Ferguson, Eldon E.; and Broida, H. P.: Charge-Transfer Absorption Spectra of $\mathrm{NO}$ in $\mathrm{Kr}$ and $\mathrm{CH}_{3} \mathrm{OH}$ Solutions. J. Chem. Phys., vol. 40, 1964, p. 3715.

31. Ferguson, E. E.; and BroidA, H. P.: A Possible Mechanism for Light Absorption by Interstellar Grains, Astrophys. J., vol. 141, 1965, p. 807.

32. Swings, P.; and Desirant, M.: Ciel et Terre-No. 5, 1939, p. 160.

33. Swings, P.; and Ohman, Y.: The Observatorv. vol. 62, 1939, p. 150.

34. Herzberg, G.: Spectra of Diatomic Molecules. Van Nostrand, 1950. 
35. Weltner, W., Jr.; Walsh, P. N.; and ANgell, C. L.: Spectroscopy of Carbon Vapor Condensed in Rare-Gas Matrices at $4^{\circ}$ and $20^{\circ}$ K. J. Chem. Phys., vol. 40, 1964, p. 5.

36. Kramers, H. A.; and Heisenberg, W.: Z.Physik., vol. 9, 1931, p. 273.

37. Goppert-Mayer. M.: Ann. Physik., vol. 9, 1931, p. 273.

38. Breit, G.: Rev. Mod. Phys., vol. 4, 1932, p. 504.

39. Placzex, G.: In Handbuch der Radiologie. (E. Marx, ed), vol. 6, pt. 2 Akademische Verlagsgesellschaft, Leipzig, Germany, 1934, p. 205.

\section{DISCUSSION}

O'Dell: Have you tried to evaluate this process quantitatively? For instance, you used the N III recombination lines which are extremely weak. You also used He II lines, which aren't at all common in H II regions.

Johnson: I know that these are very weak features, but I forgot to mention that the inverse Raman effect has been experimentally proved by someone in Canada.

O'Dell: The reason I mention this is that there are more common ions which just don't happen to fall in the region of the visible spectrum, and this very attractive mechanism may be just as well excited in the case of He I by 10830 . This is a line we do not consider in the spectrum, yet it is extremely intense in the.H II regions and there are other ultraviolet lines. I wonder if perhaps you could present an even stronger argument if you include lines that you would expect to observe rather than just those that are?

Johnson: This is a good point. If these are indeed vibrational frequencies, you should get some of the higher overtones that would then take you into the UV; so perhaps one should look into the UV region.

Wickramasinghe: What is the number density or mass density that you require to produce the observed strengths of the interstellar lines?

Johnson: For mechanism two, I feel confident that there is enough strength to account for the interstellar lines because the $f$-values for unpaired electrons are quite high for these transitions.

Stecher: Do you have any estimate of the cross section? If you take diatomic molecules, such as $\mathrm{H}_{2}$, the Raman scattering has a very small cross section, about 1 percent that of Rayleigh scattering.

Johnson: What $I$ am doing is extrapolating the experimental results. There is a very small probability that the inverse Raman effect will occur. In fact, no one had seen it with incoherent light until the advent of lasers. It needed this high-intensity laser flash, plus a Stokes-shifted Raman line to give a continuum occurring at the same time. I tried to get the inverse Raman effect by using a flash lamp and a laser lamp and it was very difficult to do experimentally because the two pulses must occur at the same time or ordinarily just blackening of the plate results. 
However, I am doing a lot of coherent Raman scattering work with lasers and I have recently been able to get the inverse Raman effect.

Donn: I think that the set of alternatives proposed here is a very exciting and stimulating argument for looking at the chemical theory of interstellar matter. It is a very constructive hypothesis, for it suggests various things that may be involved. One point of interest is that the basis of Wickramasinghe's suggestions for grains, various molecules may form and be ejected from these red giant stars. In fact, as calculations that have been made by Bauer and by Tsuji show, the atmospheres for these red giant stars contain many species of molecules of the kind suggested by Johnson. If this mechanism of mass exchange takes place through these cool stars, we may be feeding many such molecules into interstellar space, as Tsuji has already pointed out. This suggests that we may be able to tie together several features of interstellar matter.

With regard to the Raman scattering, I think that the coincidences are interesting, but because of the small cross sections for Raman scattering and the larger cross section for Rayleigh scattering, it would seem that if enough material were in space to give the Raman scattering for these lines, Rayleigh scattering would then give a significant contribution to interstellar extinction.

Greenberg: How many molecules would be required per unit of volume?

Johnson: I can only give you preliminary estimates. At the moment it doesn't look optimistic, but I don't have good numbers for the intensities of these emission features.

Dressler: In answer to Dr. Greenberg's question, I think I have given the relevant numbers in my paper. For instance, in the $4430-\AA$ band the number of absorbing molecules times the $f$-value is on the order of $10^{-8} / \mathrm{cm}^{3}$. When you compare that with a classical type of grain density, you get something like $10^{-5}$ absorbing molecules per bulk molecule of the grain. If these molecules are assumed to be in the grains and $10^{-3}$ is assumed as the $f$-value for that type of transition in the visible, it suffices to have a concentration of about 1 percent of the absorbing type of molecule in the grain. In other words, it is not necessary to have grains existing exclusively of these absorbing molecules.

Nandy: Your mechanism requires the presence of an emission line. If that emission has to be produced by the star, then $4430 \AA$ should be present in the spectra of only those stars which show emission lines. Have you checked that?

Johnson: I have not done this. However, there are much more fundamental difficulties with the inverse Raman type than these astronomical ones; namely, you always need an emission line and you also have to start off with an anti-Stokes line. For some of the ones I showed 
there are none of these anti-Stokes emission features, so it can be ruled out on that basis alone. But maybe I don't see the whole picture. Is it not correct that some observations have been made on the polarization of the $4430-\AA$ feature?

Wampler: We now have a theory that we can check rather easily and observations can be obtained rather quickly. I am curious as to why you have only 24 lines; why don't you have a thousand lines in the spectrum? I would think there would be an enormous number of different types of simple molecules, and each of these would have two or three lines and very quickly the entire spectrum would be filled up.

Johnson: Maybe it is a question of strength. I picked out the ones which were strongest.

W. E. Thompson: It should be pointed out that when you come to the stage of attempting to identify carriers of these features, you need to consider the vibrational frequencies of the electronically excited molecules, not the ground state molecules. I expect that most of the molecular vibrational frequencies you have quoted were obtained by infrared or Raman measurements, and hence apply to the ground electronic state. They will be different in the excited state.

Johnson: That's a good point. These are for the excited state.

Walker: The inverse Raman effect is also an efficient scatterer of the appropriate emission line itself. Thus for the helium emission lines, for example, it is quite possible that we wouldn't detect an emission line of appreciable intensity. But the more serious criticism is that there is no absorption band associated with the Balmer series.

Johnson: But I threw out the Balmer series. I looked at $\mathrm{H} \alpha, \mathrm{H} \beta, \mathrm{H} \gamma$, etc., and I threw them out because I didn't see any pairing. I needed two emission lines; so I used $\mathrm{H} \alpha$ in conjunction with all the other emission lines and I could not get any sets. Maybe this is the wrong criterion to use. On the other hand, $\mathrm{H} \alpha$ is pretty far in the red and some emission lines farther in the blue region are desirable.

Field: In your second hypothesis you can predict whether the energy absorbed by the molecule will be accommodated to the solid or whether it will be immediately reemitted at the same wavelength, thus producing a scattering which might be observed.

Johnson: These features that you pointed out I think are worth investigating in detail. 